\title{
Entre posible y lo deseable: los proyectos topográficos geodésicos de la Argentina (1912-1950)
}

\section{BETWEEN POSSIBILITY AND DESIRE: GEODESIC TOPOGRAPHIC PROJECTS IN ARGENTINA (1912-1950)}

\author{
ENTRE POSSÍVEL E O DESEJÁVEL: OS PROJETOS TOPOGRÁFICOS \\ GEODÉSICOS DA ARGENTINA (1912-1950)
}

\begin{abstract}
Malena Mazzitelli Mastricchio
Para citar este artículo: Mazzitelli Mastricchio, M. (2019). Entre posible y lo deseable: los proyectos topográficos geodésicos de la Argentina (1912-1950). Perspectiva Geográfica, 24(2), 35-48. doi: $10.19053 / 01233769.8494$
\end{abstract}

Recepción:

15 de octubre de 2018

Evaluación:

9 de mayo de 2019

Aprobación:

29 de mayo de 2019

\section{Resumen}

Desde 1912, el Instituto Geográfico Militar (IGM) propuso un plan cartográfico nacional que implicaba mediciones geodésicas, tanto de tipo altimétrico como para la medición de las coordenadas geográficas. El proyecto se consideraba superador respecto de otra clase de mediciones existentes. Sin embargo, el plan del IGM fue muy ambicioso y, a pesar de haber sido readaptado en varias oportunidades, hubo que esperar hasta fines de la década de 1940 para cumplir con los propósitos. El objetivo de este trabajo es reconstruir las prácticas desplegadas por la institución para desarrollar dicho plan. Para ello, se usaron las publicaciones realizadas por el IGM en el momento en que se presentaron los planes cartográficos. El análisis permitió concluir que los proyectos superaban no solo las expectativas, sino también las posibilidades técnicas con las que contaba el país para realizar esa clase de mediciones.

Palabras clave: Argentina, geodesia, plan cartográfico, topografia.

1 Doctora en Geografía. Facultad de Humanidades y Ciencias de la Educación de la Universidad Nacional de La Plata, Argentina. mastricchiomalena@gmail.com. 


\section{Abstract}

In 1912, the Military Geographical Institute (IGM) proposed a national cartographic plan that involved geodesic measurements, both of an altimetric type and for geographical coordinates. The project was considered to be superior in relation to other classes of existing measurements. Still, the IGM plan was overly ambitious and, despite having been re-adapted several times, it was necessary to wait until the end of the 1940s to fulfill its purposes. The objective of this paper is to reconstruct the practices followed by the institution in developing such plan. To this end, IGM documents released upon introduction of the cartographic plans were used. It is concluded that the projects exceeded not only the expectations, but also the technical possibilities that the country had to take these measurements.

Keywords: Argentina, geodesy, cartographic plan, topography.

\section{Resumo}

Desde 1912, o Instituto Geográfico Militar (IGM) propõe um plano cartográfico nacional que envolve medições geodésicas, tanto do tipo altimétrico quanto para a medição das coordenadas geográficas. O projeto considerava-se superador respeito de outra classe de medições existentes. No entanto, o plano do IGM foi muito ambicioso e, apesar de ter sido readaptado em várias ocasiões, era necessário esperar até o final da década de 1940 para cumprir os objetivos. O propósito deste trabalho é reconstruir as práticas empregadas pela instituição para desenvolver o referido plano. Para isso, foram utilizadas as publicações feitas pelo IGM no momento da apresentação dos planos cartográficos. A análise permitiu concluir que os projetos excederam não somente as expectativas, mas também as possibilidades técnicas com as quais o país teve para realizar esse tipo de medições.

Palavras chave: Argentina, geodésia, plano cartográfico, topografia. 


\section{Introducción}

En 1912, el Instituto Geográfico Militar (IGM) (1904-2009) presentó su proyecto cartográfico de largo alcance, que se denominó Plan de la Carta. Este plan tenía varios objetivos, entre ellos, dotar al país de una cartografía con precisión geodésica a escalas diferentes ${ }^{2}$, y otra de las metas que se propuso a través del plan fue trazar una red de puntos geodésicos por todo el país. Para llevar a cabo este plan cartográfico, el IGM puso en marchas distintas estrategias que le permitieron colocarse a la vanguardia de los trabajos cartográficos nacionales y estar al nivel de las exigencias internacionales.

En este artículo intentamos reconstruir las prácticas desplegadas por el Instituto Geográfico Militar para que el país contara con mediciones geodésicas. Para ello repasamos, primero, los aspectos centrales de la ciencia geodésica, y a continuación presentamos las etapas y modalidades en que se desarrolló la red geodésica horizontal argentina, así como las diversas dificultades que retrasaron los trabajos. En la segunda parte se describen los avances en la nivelación que se realizaron en la Argentina a fines del siglo XIX y los propuestos por el IGM en el siglo XX.

\section{Una ciencia geodésica}

Hablar de mediciones con precisión geodésica implica que los valores de las coordenadas geográficas y los valores de las alturas están referidos a una

2 Las escalas serían: 1:25.000 para mapas de interés militar; 1:100.000 para la cartografía civil; $1: 1.000 .000$ para las hojas que formaban parte del proyecto cartográfico mundial conocido como Mapa Millonésimo, y el mapa mural del país a escala 1:2.000.000 (sobre el tema véase Mazzitelli Mastricchio, 2008)

Vol. 24 N. ${ }^{\circ} 2$

julio - diciembre de 2019

pp. $35-48$ figura geométrica. Una de estas figuras es conocida como geoide. Carl Friedrich Gauss señaló en 1828 que había llegado a concebir una nueva superficie de referencia, a la que él mismo definió en los siguientes términos: "lo que llamamos la superficie de la Tierra en el sentido geométrico no es más que esa superficie que intersecta en todos los lados la dirección de la gravedad en ángulos rectos, y parte de la cual coincide con la superficie de los océanos" (citado en Ruiz y Ruiz, 2000, p. 209). Pero no fue sino hasta 1873 que Johann Benedict Listing (1808-1882) acuñó el término geoide por primera vez. Durante cien años, la determinación del geoide fue la meta principal de la geodesia (Ruiz y Ruiz, 2000, p. 210). En otras palabras, el geoide es la superficie suavizada, pero irregular, que tendrían los océanos si se los extendiera por debajo de la superficie continental, manteniendo solo como causa de movimiento la gravedad de la Tierra y la rotación terrestre. El elipsoide de revolución es una figura matemática a la cual debe adaptarse lo mejor posible el geoide. Utilizar estas figuras como base para las mediciones es lo que se conoce como un sistema de referencia geodésico. Para construir este sistema de referencia es preciso seleccionar un punto en la superficie terrestre, para el cual se calculan valores de latitud y longitud, de altura elipsoidal (la altura con referencia al elipsoide), geodésica (la cota con referencia al geoide), y acimut (o ángulo que le da orientación al elipsoide). Lo anterior es necesario porque para definir un elipsoide, además de calcular el valor del semieje mayor o ecuatorial y del semieje menor o polar, se lo debe orientar respecto a la Tierra real. La recta normal al elipsoide y la recta normal al geoide coinciden en el datum, que representa el origen del sistema. Todas las mediciones, por lo tanto, van a estar referidas al datum, de manera tal que cualquier cambio en el mismo modifica todo el sistema. Debido a esto, las 
coordenadas que fueron calculadas con un mismo datum pueden ser relacionadas entre sí (Figura 1).

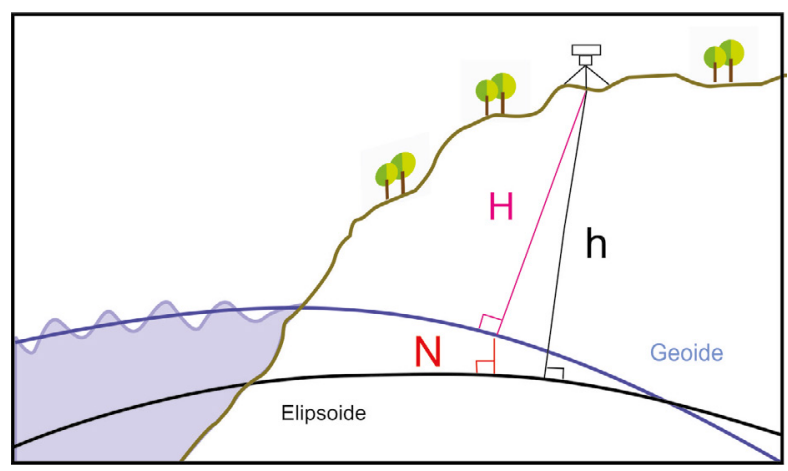

FIGURA 1.

Esquema de geoide y elipsoide: la altura ortométrica o topográfica está representada en la $\mathrm{H}$; la altura al elipsoide se representa con la $\mathrm{h}$; la letra $\mathrm{N}$ es la atura geoidal, de modo que $\mathrm{h}=\mathrm{H}+\mathrm{N}$.

Fuente: Elaboración propia.

Con relación a las alturas existen, al menos, dos tipos: la altura ortométrica y la elipsoidal. La primera es la distancia que existe desde la superficie de la Tierra al geoide (identificada con la letra $\mathrm{H}$ ); esta es conocida como la altura topográfica. La segunda, la altura elipsoidal, es la distancia que existe desde la superficie de la Tierra al elipsoide (identificada con la letra h) (Figura 1). Como entre ellas existen diferencias (relacionadas con la gravedad), es necesario realizar las correcciones: $\mathrm{h}=\mathrm{H}+\mathrm{N}$, siendo $\mathrm{N}$ la distancia entre elipsoide y geoide ${ }^{3}$.

3 Uno de los cálculos necesarios para elegir un elipsoide adecuado al geoide es la desviación de la vertical. Las dos características más importantes del geoide son: en primer lugar, que el potencial gravimétrico sea el mismo en todas sus partes, y en segundo lugar, que la dirección de la gravedad sea siempre perpendicular al geoide. Pero como la superficie del elipsoide es regular y la del geoide es irregular, en la intersección de ambas superficies se forma un ángulo, denominado desviación de la vertical del punto. Esta desviación es causada por el excedente de las masas montañosas y la diferencia con las masas oceánicas. La dirección de la plomada es atraída por la masa montañosa con respecto al elipsoide; de forma contraria, la deficiencia de masa en el océano empuja la dirección de la plomada, por lo tanto, su dirección es siempre perpendicular al geoide. El geoide queda, de esta manera, por encima del elipsoide en zonas
Es sabido que, desde mediados del siglo XIX, gran parte de los Estados modernos occidentales habían cartografiado o, cuanto menos, planificaban cartografiar sus territorios bajo el método de estas modernas técnicas (Nadal y Urteaga, 1990; Ruiz y Ruiz, 2000; Thrower, 2002). Los desarrollos de las técnicas aplicadas a la cartografía, a la geodesia y a la topografía estaban suficientemente avanzados en los países europeos y en Estados Unidos, y permitía plantear cuestiones tales como la medición de la gravedad, la trigonometría, la elección del geoide, las mediciones de arcos de meridiano, la adopción del sistema métrico y un único meridiano de referencia.

En este contexto científico, los profesionales y técnicos argentinos de la mensura buscaron construir una cartografía geodésica para el territorio nacional ya que, si querían ser partícipes de las discusiones internacionales, debían presentar proyectos de relevamiento territorial acordes a las exigencias técnico-científicas de la época, aunque en muchos casos las instituciones no contaran con el capital financiero ni humano necesarios para cumplir en tiempo y forma los objetivos propuestos.

\section{Red geodésica horizontal}

En la Argentina, a principios del siglo XX, se contaba con algunas mediciones trigonométricas realizadas de forma aislada por el Instituto Geográfico Militar. El objetivo de estas mediciones era para dotar de puntos conocidos a algunas planchetas ${ }^{4}$,

montañosas y por debajo en los océanos (Caire Lomelí, 2002, p. 21).

$4 \quad$ La plancheta es un instrumento topográfico que permite ir dibujando el mapa en el campo mismo, y está compuesto por un trípode que sostiene una base donde se realiza el dibujo. La base tiene movimientos, de tal manera que permite dirigir visuales a diferentes puntos. Esta metodología 
pero no alcanzaban a constituir una red geodésica. Por ejemplo, el primer ensayo de una triangulación geodésica fue entre 1900 y 1902 en la provincia de Mendoza, donde se midieron en total 2.600 metros. Este trabajo se realizó con cinta invar de 50 metros, cuyo material no se dilataba con las temperaturas, por lo que daba como resultado una medición más precisa (IGM, 1953). De hecho, el instituto recupera varios de estos trabajos ejecutados de forma aislada y con referencias geodésicas locales, que se realizaron entre los años 1900 y 1912, como antecedentes para el trabajo geodésico general que se propone en 1912. Un ejemplo es la triangulación, en 1909, en la zona militar de Campo de Mayo, cercana a la ciudad de Buenos Aires. La recopilación de estos antecedentes por parte de la institución era parte de la estrategia del IGM para construir su propia tradición en asuntos cartográficos y posicionarse como única institución técnica de la Argentina capaz de realizar estos trabajos (Lois, 2004), objetivo que logró en $1941^{5}$. Sin embargo, la red geodésica integral se planificó a partir de la presentación que el IGM hizo en el Plan de la Carta de la República, en 1912.

El plan formulado en ese año proponía construir cadenas de triángulos de primer orden cuyos lados debían tener una longitud media de 40 a 60 $\mathrm{km}$ en terrenos llanos, dimensión que cambiaba a medida que el relieve se hacía más accidentado. Por ejemplo, en terrenos con relieves más suaves y ondulados, donde la distancia era más estable y constante, podía ser de $20 \mathrm{~km}$; pero en zonas cuyo

\footnotetext{
de levantamiento fue ampliamente usada en la Argentina para elaborar la cartografía topográfica del territorio nacional durante las primeras décadas del siglo XX.

5 En 1941 se sancionó la Ley $n^{\circ}$ 12.696, conocida como Ley de la Carta, que otorgaba, entre otras cosas, al Instituto Geográfico Militar el monopolio de la representación del territorio nacional. Véase Lois y Mazzitelli Mastricchio (2009).
}

Vol. 24 N. ${ }^{\circ} 2$

julio - diciembre de 2019

pp. $35-48$ relieve era más abrupto y las montañas alcanzaban una altura suficiente como para dificultar la visual que se necesitaba para realizar la medición, la distancia debía ser modificada de acuerdo con las necesidades de cada lugar. La red tenía que seguir los rumbos principales de norte a sur y de este a oeste $\mathrm{y}$, en la medida de lo posible, la costa marítima y los límites internacionales e interprovinciales, "a fin de facilitar tanto la determinación exacta de las áreas, como los límites de la República y de cada provincia o territorio" (IGM, 1951, p. 74). Los instrumentos que se usarían para realizar el trabajo serían el teodolito y cintas y alambres de aleación invar, un material que no sufren dilatación y da como resultado una medida más estable.

La Figura 2 muestra el proyecto y la traza por donde debía pasar la red de triangulación del territorio argentino. A pesar de que en la leyenda que acompaña el mapa se propone un tiempo de realización de 25 años, el plan fue algo ambicioso y, como veremos, tuvo que ser modificado a lo largo de los años para adaptarlo a las condiciones técnicas y económicas del país. Por una parte, en 1912 (cuando se presentó el plan) el plantel estable del instituto no contaba con la suficiente experiencia técnica, ni tampoco con la cantidad de personal suficiente (un total de 30 técnicos) para encarar una obra de tal magnitud ${ }^{6}$. Por otro lado, el IGM hace mención

6 El 1904 el IGM contaba con un total de 33 técnicos, así: dos escribientes; cuatro ingenieros civiles; dos cartógrafos; un mecánico de precisión; dos litógrafos; dos dibujantes calígrafos; cuatro dibujantes topógrafos; dos fotógrafos: dos computadores; dos impresores; un maquinista; cuatro ayudantes; dos ayudantes de fotógrafos; dos aprendices de dibujo y un aprendiz de mecánico. Cinco años después de que haya presentado el plan, el IGM incrementó su personal a 50 técnicos: incorporó a cuatro cartógrafos; un mecánico de precisión; 14 dibujantes topógrafos; 17 geodestas; un geodesta astrónomo; diez topógrafos y tres calculistas. Si bien es cierto que el número amentó considerablemente, sobre todo el de los geodestas, no es una cantidad suficiente para llevar a cabo la empresa que se proponían, por lo menos no en el lapso de tiempo de 25 años. 
a que ciertas limitaciones financieras comprometían los planes de trabajo; en el primer Anuario publicado por el Instituto Geográfico Militar, en 1912, se reconoce la dedicación a tareas exclusivamente militares de los trabajos cartográficos y se alude a motivos económicos: "Los exiguos recursos y elementos escasos con que fue dotada [la repartición], eran apenas suficientes para responder a las necesidades apremiantes: levantamientos con propósitos militares, estudios en las fronteras, cuestiones de límites, cartas y planos para maniobras del Ejército" (IGM, 1912, p. 6).

Los trabajos geodésicos, por lo tanto, no fueron ajenos a la cuestión económica, y también se resintieron y se modificaron varios de los objetivos propuestos. En primer lugar, a principios de la década de 1920 se produjo un redireccionamiento en la red y la triangulación principal. El trazado no se haría siguiendo los límites internacionales e interprovinciales como había sido planteado por el IGM en 1912, sino que sería desarrollado a lo largo de los paralelos y meridianos, formando "cuadriláteros de $2^{\circ}$ de latitud por $2^{\circ}$ de longitud" (IGM, 1951, p. 76). El tamaño de los triángulos, que antes se adaptaba a la visibilidad del terreno, ahora quedaba homogeneizado y estandarizado para todos los tipos de relieve.

Por otro lado, en 1916 se creó la Comisión Astronómica Expeditiva, cuyo fin era proporcionar coordenadas astronómicas al IGM. Estas coordenadas tienen la ventaja de que pueden ser medidas independientemente del elipsoide elegido, sin que esto intervenga en su precisión. Esto es así porque la vertical astronómica o vertical del lugar (a

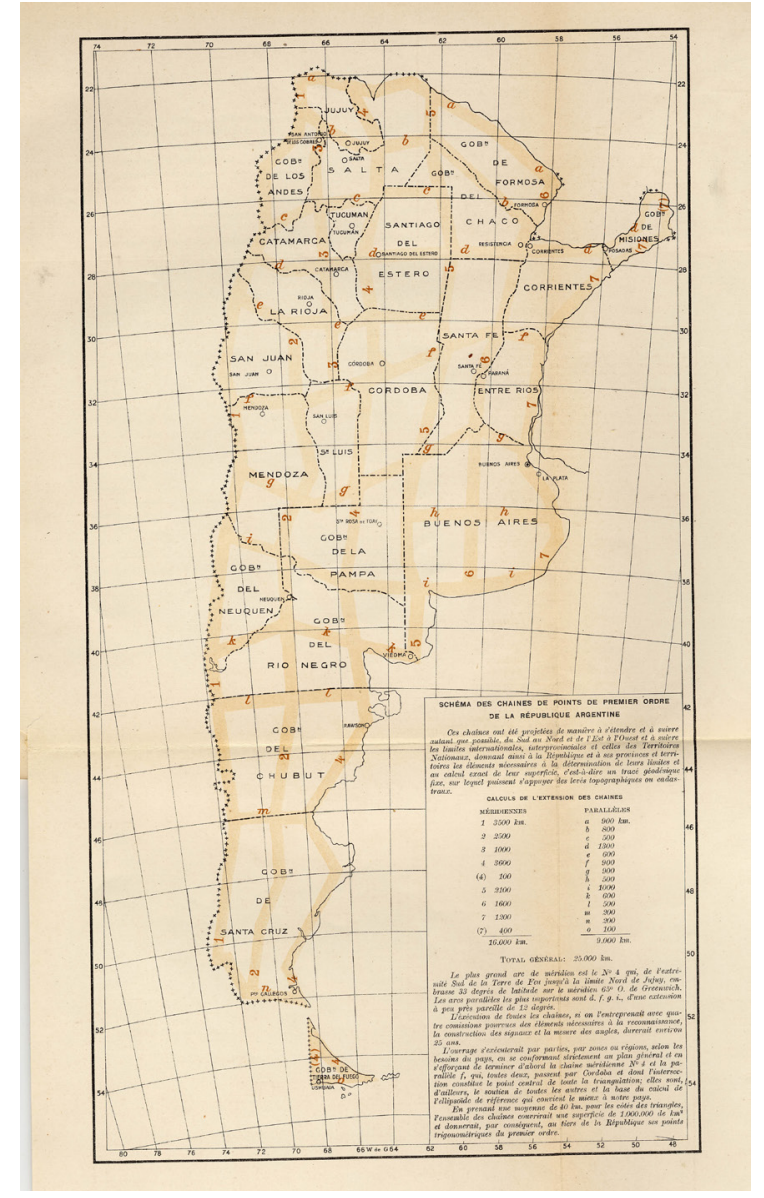

FIGURA 2.

Proyecto y traza de la red de triangulación. El texto que acompaña la imagen dice: "El arco de meridiano más extenso es el 4 que desde la punta de Tierra del Fuego hasta el límite Norte de Jujuy, encierra $33^{\circ}$ de latitud del meridiano $65^{\circ}$ o. de Greenwich. Los arcos paralelos más importante son cuatro (d. j. g. i) de extensión semejante, igual a $12^{\circ}$ aproximadamente. La ejecución de todas las cadenas, si se emprendiese con cuatro comisiones provistas de los elementos necesarios para el reconocimiento, construcción de señales y medición de ángulos, duraría más o menos 25 años".

Fuente: IGM (1912).

partir de la cual es posible determinar la latitud y la longitud astronómica) ${ }^{7}$ es la línea que toma la

$7 \quad$ La latitud astronómica es el ángulo que se forma entre la vertical del lugar y el plano ecuatorial. La longitud astronómica, en cambio, es el ángulo formado por el meridiano astronómico local y el meridiano de origen. El meridiano 
plomada en un punto determinado en función del campo gravitatorio y es independiente del elipsoide adoptado (Franco Rey, s. f.). Algunos autores sostienen que una manera eficiente de seguir con los trabajos topográficos y geodésicos, a pesar de la crisis financiera que los afectaba, era realizar coordenadas astronómicas. Por ejemplo, Raymond Craib sostiene, para el caso mexicano, que entre las consecuencias que acarrearon los problemas financieros estuvo el abandono de las observaciones geodésicas y la adopción de métodos astronómicos para realizar los trabajos de campo y para determinar la ubicación geográfica de los puntos (Craib, 2000, p. 145).

En la Argentina, si bien los puntos medidos por la comisión estarían distribuidos por todo el territorio de la república, los primeros trabajos se realizaron en la zona del río de la Plata siguiendo las vías ferroviarias. De este modo, se dotó de valores de latitud y longitud a los empalmes ferroviarios. A su vez, el acimut era medido con un teodolito, con el cual se determinaba la dirección de alguna recta (que por lo general era la recta del ferrocarril). Esta recta solo tenía por objetivo ayudar a transportar las estaciones (puntos con datos conocidos) a los centros de las plazas, las iglesias o algún otro edificio que asegurara la visibilidad de los puntos. La red ferroviaria de Argentina, que para 1910 tenía una extensión de 27.994 km, sirvió como columna vertebral para los trabajos cartográficos; esto incluye la medición de las alturas y los valores horizontales de latitud y longitud.

A partir de 1923 se adoptó el elipsoide Hayford para los cálculos geodésicos, tal como proponía la Unión Geodésica y Geofísica Internacional, y se

astronómico es el plano que pasa por la vertical astronómica y el punto medido (Franco Rey, s. f.). abandonó el de Bessel, propuesto en 1912 (IGM, 1912, p. 8). A pesar de la crisis político-económica que atravesaba la Argentina ${ }^{8}$, en la década de 1930 se produjeron grandes avances en trabajos de tipo geodésico: se calculó la medición de los vértices de diferentes cerros, se realizaron trabajos de medición en el sur de la gobernación del Neuquén, en Buenos Aires, en el delta del Paraná y regiones adyacentes, y en la provincia de Entre Ríos se efectuó una triangulación que abarcó una superficie de $6.500 \mathrm{~km}^{2}$.

En 1936 se conformó la Comisión para la Medición del Arco de Meridiano, que tenía entre sus funciones la determinación del planteamiento estratégico de la campaña geodésica y la formación del personal técnico y auxiliar de campaña. También se ocupaba de la administración y el mantenimiento de los vehículos, del instrumental y de los medios para la subsistencia del personal durante la campaña (esto es, alojamiento temporal y alimentación, etcétera). En cuanto a los trabajos desarrollados en gabinete, la comisión estaba encargada de formar un archivo con los datos, facilitar el "procesamiento numérico regular de los datos provenientes de la campaña, y plantear el estudio estadístico de los errores de medición, de los que en alguna medida dependía el control y el buen éxito de las mediciones de campaña" (Ortiz, 2005, p. 109). A su vez,

8 Durante los años 1930-1943 la Argentina atravesó cambios políticos importantes, como golpes de Estado y fraudes electorales que llevaron a que este periodo sea conocido por los historiadores como la década infame o la restauración conservadora. Asimismo, la crisis económica mundial de 1929 impactó fuertemente en la economía del país produciendo una baja importante en las exportaciones: "el conjunto de los precios de exportaciones de la Argentina era en 1932 el 37\% de los precios de 1928" (Saborido y de Privitellio, 2006: 210). Asimismo, durante este período se sentaron las bases para el inicio de la industrialización como consecuencia de las dificultades experimentadas con el comercio exterior y la disminución de las exportaciones e importaciones (Saborido y de Privitellio, 2006). 
el personal de alto rango ${ }^{9}$ estaba encargado de las innovaciones científico-técnicas que pudieran mejorar las tareas de la comisión.

La comisión dependía formalmente del Ministerio de Justicia e Instrucción Pública y su presidente fue Félix Aguilar (1884-1943), quien tenía gran experiencia en trabajos geodésicos. Aguilar fue uno de los primeros astrónomos argentinos, se había recibido en 1910 de ingeniero geógrafo en la Universidad de la Plata y luego tuvo la oportunidad de perfeccionarse en Alemania, Francia e Italia. Se desempeñó como director del Observatorio de la ciudad de La Plata en dos oportunidades y fue profesor de la Escuela Superior de Guerra del Ejército, donde dictó cursos de astronomía y geodesia. Aguilar había estado a cargo de la Sección Geodesia del IGM en 1921, pero cuando fue nombrado presidente de la comisión ya no ocupaba ese cargo. A pesar de que la presidencia de la comisión quedó en manos de un científico que no era parte del personal del instituto, este apoyó y participó de los trabajos de la comisión desde un principio, aportando tanto el instrumental técnico como las instalaciones y el capital humano; además, el director del IGM era uno de los expertos que tomaban decisiones en el seno de la comisión. El instituto designó a cuatro de sus especialistas en geodesia para que se pusieran al servicio de las tareas requeridas para la misión. La partición de este personal no era para nada despreciable si se tiene en cuenta que "con este personal la Comisión pudo

9 Además de Félix Aguilar, la comisión estaba compuesta por un integrante de la Universidad de Buenos Aires, el ingeniero Eduardo Baglietto; el director del IGM, el coronel Baldomero de Biedma; un representante del Ministerio de Marina, Melchor Z. Escola; un representante del Museo de La Plata, el Dr. Joaquín Frenguelli; un integrante de la Universidad de Córdoba, el ingeniero Tezanos Pinto, y, por último, un representante de la Universidad de La Plata, el astrónomo monseñor Fortunato J. Devoto. Sobre la historia de la Comisión para la Medición del Arco de Meridiano, véase Ortiz (2005) iniciar las tareas de reconocimiento trigonométrico y de nivelación de precisión que de otro modo no hubiera podido enfrentar. Además, en los talleres del IGM se construyeron marcas, señales, torres y otros auxiliares de geodesia necesarios para la Comisión" (Ortiz, 2005, p. 115).

Paralelamente a esta comisión, el IGM seguía con sus objetivos y para esta época se pudo determinar la longitud entre Potsdam, ciudad alemana en donde se constituía el datum de referencia para los países de Europa occidental, y la ciudad de Buenos Aires. Estos cálculos eran beneficiosos para la organización del Servicio de la Hora, para la red geodésica nacional, pero sobre todo posibilitaba que la Argentina acompañara el ritmo de la ciencia cartográfica internacional.

En 1946, a través de las Disposición Permanente $\mathrm{n}^{\circ} 440$, se propuso como origen del sistema horizontal de coordenadas el punto conocido como Campo Inchauspe, ubicado en Pehuajó, en la provincia de Buenos Aires, seleccionado debido a su estabilidad magnética y geológica. Ubicado cerca de la intersección del paralelo de $36^{\circ}$ sur y el meridiano de $65^{\circ}$ oeste, fue el origen del sistema de triangulación de la Argentina, vigente durante más de cincuenta años. La cartografía sistemática de instituciones nacionales, como la Dirección Nacional de Minas, Geología e Hidrología ${ }^{10}$, utilizaba los cálculos del IGM y sus valores de medición estaban relacionados.

A pesar de los esfuerzos, la homogenización de las coordenadas llevó un tiempo largo. En 1948, el instituto publicó un libro llamado Coordenadas

\footnotetext{
10 Las hojas topográficas impresas de la Dirección Nacional de Minas no tienen coordenadas planas; sin embargo, los originales de campo levantados a escala 1:100.000, manuscritos realizados por los topógrafos, sí cuentan con este tipo de información.
} 
provinciales, geográficas y planas conformes de puntos trigonométricos de I y II orden. En el prólogo, escrito por el entonces director del IGM, el general Otto Helbing (1892-1968) -el mismo que tomó la decisión de colocar como origen del sistema Campo Inchauspe-, reconoce los distintos sistemas que todavía convivían en la Argentina. Al parecer se superpusieron distintos sistemas de referencia geodésicos provinciales, por ejemplo: en la provincia de Santa Fe las coordenadas se medían a partir de un punto astronómico de carácter expeditivo en Paraná, y la red geodésica entrerriana se apoyaba en un punto ubicado en Ubajay. En la provincia de Corrientes, las mediciones trigonométricas realizadas después de 1932 partieron de un punto astronómico colocado en Iraembe-Miní. En San Juan, la cadena de red geodésica, medida con dirección norte-sur, partía del punto astronómico ubicado en Chos Malal. La provincia de Mendoza, durante el período 1928-1936, apoyó sus mediciones astronómicas en la cadena de San Juan. Muchos de estos puntos se habían referenciado al elipsoide de Bessel y luego fueron recalculados según el propuesto en 1923. El citado libro de Coordenadas provinciales (IGM, 1948) tenía la intención de ofrecer esta información para que los usuarios pudieran adaptar los cálculos al sistema correspondiente. Una posible explicación de la convivencia de estos diferentes sistemas provinciales puede deberse a que el IGM, para paliar la crisis financiera y la falta de financiamiento por parte del Gobierno nacional, realizó varios convenios con Gobiernos provinciales, con el fin de realizar "trabajos astronómicos, geodésicos y topográficos necesarios para la obra de catastro y el mapa de [cada] provincia" (IGM, 1919, p. 235). Los acuerdos consistían en que el IGM realizaba mediciones geodésicas que le permitirían a las provincias obtener el mapa catastral de su territorio; a cambio, el IGM recibía financiación y podía disponer de los datos obtenidos y usarlos para concluir el plan original ${ }^{11}$.

En la Argentina, las redes geodésicas mantenían por separado el marco de referencia horizontal del vertical, es decir, la red horizontal de coordenadas partía de un origen diferente al del sistema de referencia vertical: mientras el primero lo hacía de Campo Inchauspe, el segundo, como veremos, se asocia al PERN ubicado en Tandil.

\section{Red geodésica vertical}

La primera centralización altimétrica de la Argentina se produjo en 1899, cuando el Ministerio de Obras Públicas (MOP) determinó que todas las mediciones altimétricas debían referirse al nivel cero del Riachuelo. Allí se debían remitir todas las mediciones altimétricas, por ejemplo, las utilizadas para calcular el trazado de los ferrocarriles y la altura de sus estaciones, y también para la cartografía. La decisión de unificar las mediciones al nivel cero del Riachuelo no fue azarosa, sino que se tomó porque el 17 de junio de ese mismo año se había decidido comenzar las mediciones de la primera red de nivelación realizada en el país, tarea finalizada el 28 de febrero de 1911, según el Boletín de Obras Públicas (MOP, 1912, p. 58). En este contexto, contar con un cero único resultaba fundamental para llevar a cabo la nivelación.

La nivelación estuvo a cargo de la Dirección General de Obras Hidráulicas que dependía del Ministerio de Obras Públicas, y su objetivo era establecer una base segura para los estudios hidrológicos de

11 Algunos de estos convenios fueron realizados con la provincia de Córdoba en 1917, con Mendoza en 1918 y con las provincias de Buenos Aires y Entre Ríos en 1919. 
los grandes ríos. Las líneas de nivelación se trazaron a través del recorrido de los ferrocarriles. El punto de referencia, llamado convencionalmente datum, utilizado para los cálculos fue, en ese momento, un punto situado a 19 metros de profundidad bajo la catedral de Buenos Aires. El datum coincidía aproximadamente con el nivel de bajas aguas ordinarias del río de la Plata, calculado en los talleres del Ministerio de Obras Públicas sobre la costa del Riachuelo. Se eligió ubicarlo en la catedral porteña porque ya existían en Buenos Aires otros ceros altimétricos referenciados al mismo sitio: uno era el medido por Obras Sanitarias de la Nación y el otro por la Dirección de Catastro de la Ciudad de Buenos Aires, ambos determinados en $1894^{12}$. El primero es un plano arbitrario que pasa a 30,479 metros (100 pies ingleses) por debajo del dibujo en forma de estrella que existe en el peristilo de la catedral, en correspondencia con la entrada central. El segundo, utilizado por Obras Sanitarias, estaba situado a 38.144 metros debajo de la misma estrella (MOP, 1912). Es decir, los "ceros" se refieren al mismo sitio geográfico, pero están situados a diferentes alturas. Esa diferencia se corregía fácilmente sumando o restando la medida correspondiente (el cero de Obras Sanitarias o de Salubridad está a 11,479 metros y el cero del Puerto a 19.144 metros).

Para realizar esta nivelación, el MOP había utilizado un nivel (construido por la casa Breithaupt, que desde 1762 se dedica a la construcción de instrumentos de alta precisión) y miras de tres metros

12 El ingeniero Carlos Pellegrini usó, para el catastro de Buenos Aires, un sistema de referencia local donde la ubicación de los puntos relevados estaba determinada por un sistema de coordenadas de letras y números, partiendo de dos ejes: la calle Federación (actual av. Rivadavia) y la calle 25 de Mayo. En este sistema de coordenadas, en 1829, el valor de la cota de la altura se medía referida al nivel de la playa del río de la Plata. Véase Favelukes (2005). de largo graduadas en ambas caras cada cuatro milímetros. La nivelación se materializaba en el terreno con la instalación de puntos fijos, visibles gracias a la colocación de placas de acero empotradas en los edificios de las estaciones de tren, en alcantarillas o en pilares construidos especialmente para tal fin. Esta placa permitía la colocación de una mira para realizar otra medición. También se construyeron pilares de cemento en los que la cota se visibilizaba con un clavo de cabeza esférica $y$, de este modo, complementaba las mediciones de la pared. Las placas de acero y bronce llevaban la inscripción "Nivelación de la República. MOP”.

El método empleado por el Ministerio de Obras Públicas para la medición del datum fue el geométrico. El doctor W. Seibt, a cargo del proyecto, estableció un esquema de trabajo particular: la equidistancia entre las miras siempre debía ser la misma, y el operador debía realizar la lectura con el anteojo del nivel bisectando espacios de la división menor de la mira. Las estaciones eran medidas por dos operadores; una vez determinado el desnivel, debían comprobar la lectura realizada para asegurarse de que la medición era correcta y porque todas las operaciones, salvo la corrección más fina que se realizaba en gabinete, se debían realizar en el terreno. Seguir paso a paso la metodología de medición parecía asegurar la precisión del trabajo. El Boletín de Obras Públicas de 1912 (MOP, 1912) dedica seis páginas para detallar cada pieza del nivel, entre las que se encuentra el trípode, el anteojo, el nivel brújula y los tornillos calantes, y otras tantas para explicar el método de relevamiento y adelantarse a posibles errores. Esta descripción exhaustiva del instrumental debe enmarcarse en el creciente interés por valorar las mediciones realizadas a través de instrumentos de precisión y en detrimento de las observaciones a 
partir de los sentidos.

La medición altimétrica concluida en 1911 se definía como altura topográfica, es decir, no se trataba de una medición con precisión geodésica, como el IGM empezaba a proponer. Efectivamente, solo un año después de haber terminado los levantamientos altimétricos del MOP, en donde el sistema vertical se calculaba en relación a un datum de referencia no geodésico, el IGM lanzó su propuesta cartográfica con un nuevo objetivo científico y técnico. Según el nuevo proyecto del IGM, el recorrido de las líneas de nivelación altimétrica debía seguir

[...] por donde se encontrarán mayor cantidad de puntos estables, a fin de dejar la mejor constancia de sus resultados y por donde hubiere las menores pendientes o diferencias de nivel a vencer, es decir por los caminos principales y especialmente ferrocarriles [...]. Las líneas de nivelación habrían de cerrarse del mismo modo que las cadenas de triangulación en grandes polígonos de 400 a 600 kilómetros de circunferencia (IGM, 1912, p. 22).

Pero, como ya señalamos, los objetivos de conjunto del plan formulado en 1912 eran muy ambiciosos y la determinación de la red altimétrica no fue la excepción. Tal como lo demuestra el título de la Figura 3, para 1923 la red geodésica altimétrica todavía era un proyecto. Si bien el IGM había realizado entre 1913-1915 unos puntos de nivelación aislados en la provincia de Santa Fe (IGM, 1979) ${ }^{13}$, todavía había que esperar algunas décadas más para que la Argentina contara con alturas geodésicas para todo su territorio.

13 El IGM dividió la provincia en 12 polígonos de unos 300 a 400 kilómetros de perímetro cada uno, cuyos lados se hicieron coincidir con la red de ferrocarriles (IGM, 1979).

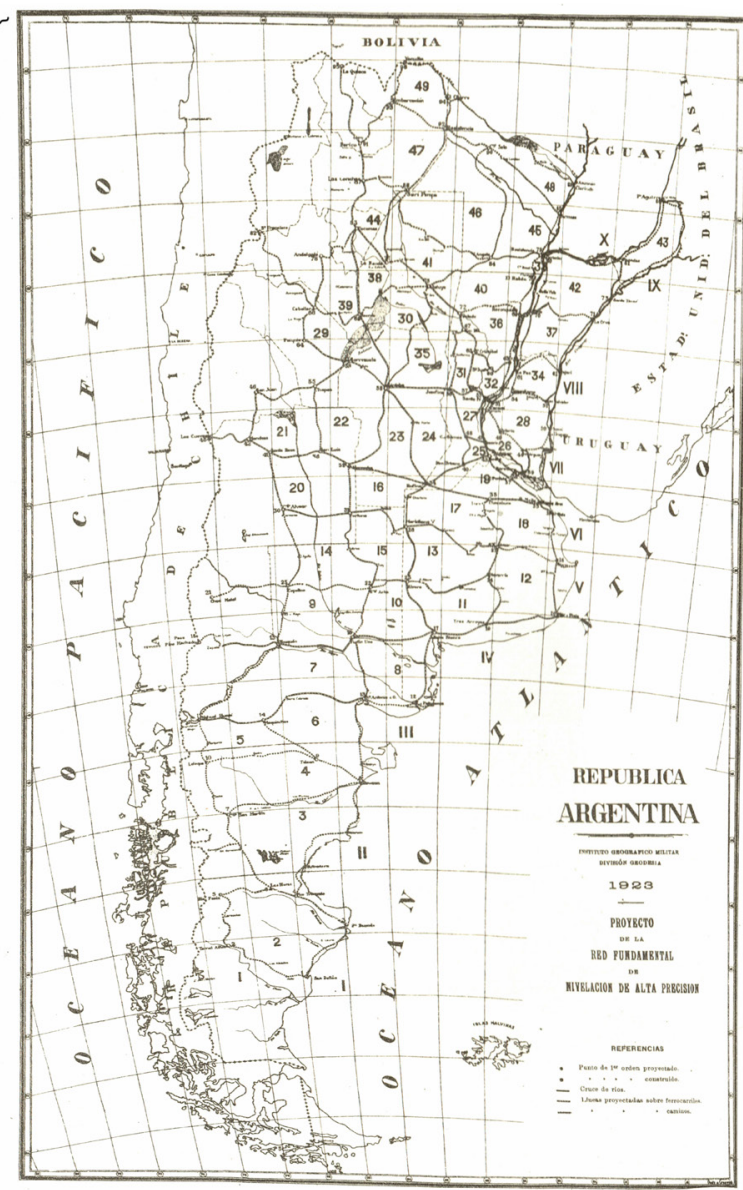

FIGURA 3.

República de Argentina. Instituto Geográfico Militar. División Geodesia. 1923. Proyecto de la Red Fundamental de Nivelación de Alta Precisión. Fuente: IGM (1979).

Recién en 1949, el IGM, de manera conjunta con la Dirección General de Minas y Geología, con Yacimientos Petrolíferos Fiscales y la Dirección del Servicio Meteorológico Nacional, calculó el punto altimétrico de referencia normal (PARN), el cual se ubicó en la ciudad de Tandil (en el Parque Independencia) por su estabilidad en relación a los movimientos y por la contextura del macizo. El PARN se conectó al mareógrafo de Mar del Plata con 75 puntos de alta precisión. Con la construcción de este datum altimétrico, las alturas en la cartografía argentina comenzaron a tener la 
precisión esperada desde principios del siglo XX. Para transportar el cero al interior del país, el IGM distribuyó una red de nivelación por todo el territorio nacional, la cual permitiría a los topógrafos realizar sus mediciones con relación al mismo cero altimétrico, independizándose de la red de ferrocarriles.

\section{Conclusiones}

A modo de cierre, podemos decir que en la Argentina de comienzos del siglo XX el IGM diseñó un plan de mediciones altimétricas y de coordenadas que buscaba una precisión geodésica y una homogenización de las mediciones cartográficas para todo el país. La materialización del proyecto llevó más tiempo que el deseado y el pronosticado por la institución. Desde el lado de la representación geodésica horizontal, coexistieron en la Argentina diferentes datum locales incluso hasta fines de la década de 1940. Para la representación altimétrica, las mediciones homogéneas empezaron con los trabajos del Ministerio de Obras Públicas publicados en 1911, que muy pronto quedaron obsoletas por no representar medidas con precisión geodésica. El IGM intentó subsanar ese defecto proponiendo un nuevo relevamiento a solo un año de haber concluido la nivelación del MOP. Sin embargo, recién a fines de la década de 1940 pudo cumplir su objetivo, y solo a partir del trabajo conjunto con otras instituciones nacionales también vinculadas a los relevamientos, como la Dirección de Nacional de Minas, Geología e Hidrología y Yacimientos Petrolíferos Fiscales.

Es decir que Argentina contó, desde principios del siglo XX, con instituciones que propusieron y diseñaron planes cartográficos generales acordes a los parámetros científicos internacionales (mediciones geodésicas, instrumental de precisión adecuado, etc.); sin embargo, implementarlos no fue una tarea sencilla y muchas veces hubo que conformarse con mediciones adaptadas a sus posibilidades técnicas y financieras. Dicho en otras palabras, los objetivos que se planificaban eran alcanzables en el papel y en la teoría; no obstante, llevarlos a la práctica implicaba afrontar otro tipo de obstáculos: en primer lugar, había que enfrentarse con un terreno bastante hostil; en segundo lugar, se contaba con pocos profesionales capacitados para llevar a cabo las mediciones; por último, las dificultades económicas y el poco presupuesto destinado a la repartición demoraron las tareas.

Incluso en el caso de las mediciones geodésicas, los trabajos llevaron mucho más tiempo del esperado y en algunas oportunidades, durante el transcurso de las mediciones, hubo que adaptarlas a nuevas exigencias científicas internacionales. Todas estas cuestiones hacían del proyecto cartográfico propuesto un plan deseable, pero imposible de alcanzar, al menos en los plazos propuestos y con los recursos disponibles. 


\section{Referencias}

Caire Lomelí, J. (2002). Cartografía básica. Ciudad de México: UNAM.

Craib, R. (2000). El discurso cartográfico en el México del porfiriato. En H. Mendoza Vargas (comp.), México a través de los Mapas (pp. 98-120). Ciudad de México: UNAM, Plaza y Valdés.

Favelukes, G. (2005). El plano de la ciudad. Expansión y control urbano en la modernización temprana de Buenos Aires (1760-1870). (Tesis de doctorado). Facultad de Filosofía y Letras, Universidad de Buenos Aires, CABA.

Lois, C. (2004). La invención de la tradición cartográfica. Litorales. Teoría, método y técnica en geografía y otras ciencias sociales, 4. Recuperado de http://www.litorales. filo.uba.ar.

Lois, C. y Mazzitelli Mastricchio, M. (2009). Una historia de la cartografía argentina. En IGM 130 años IGN, 1879-2009 (pp. 56-105). Buenos Aires: Consejo Nacional de Investigaciones Científicas y Técnicas (Conicet).

Mazzitelli Mastricchio, M. (2008). "La Carta de la República”: el programa cartográfico del Instituto Geográfico Militar (1904-1941). En C. Lorenzano (ed.), Historias de la ciencia argentina III (pp. 201-211). Buenos Aires: Eduntref.

Nadal, F. y Urteaga, L. (1990). Cartografía y Estado. Los mapas topográficos nacionales y la estadística en el siglo XIX. Geo-Crítica, 88, 7-93.

Ortiz, E. (2005). El debate de la Comisión del Arco del Meridiano: 1936-1943. En C. Lorenzano (ed.). Historias de la ciencia argentina II (pp. 28-41). Buenos Aires: Eduntref.

Rey, J. F. (S. f.). Tratado de topografía y geodesia. (Iccp) (sin datos).

Ruiz Morales, M. y Ruiz Bustos, M. (2000). Formas y dimensiones de la tierra: sintesis y evolución histórica. Barcelona: Ediciones del Serbal.

Saborido, J. y de Privitellio, L. (2006). Breve historia de la Argentina. Buenos Aires: Alianza editorial.

Thrower, N., J., W. (2002). Mapas y civilización. Historia de la cartografía en su contexto cultural y social. Barcelona: Ediciones del Serbal.

\section{Fuentes}

Instituto Geográfico Militar (IGM). (1912-1951). Anuario del Instituto Geográfico Militar de la República Argentina. Varios tomos. Buenos Aires: IGM.

Instituto Geográfico Militar (IGM). (1948). Coordenadas provinciales, geográficas y planas conformes de puntos trigonométricos de I y II orden. Sistema Castelli. Buenos Aires: IGM.

Instituto Geográfico Militar (IGM). (1951). Reseña histórica del Instituto Geográfico Militar. Su misión y su obra. Buenos Aires: IGM. 
Instituto Geográfico Militar (IGM). (1979). 100 años en el quehacer cartográfico del país (1879-1979). Buenos Aires: IGM.

Instituto Geográfico Militar (IGM). (S. f.). Curso técnico del Servicio Geográfico (Personal subalterno). Buenos Aires: IGM.

Ministerio de Obras Públicas (MOP). (1912). Anuario de 1912. Buenos Aires: Talleres Gráficos del Ministerio de Obras Públicas. 